\title{
Neutrino Mass Matrix with Approximate Flavor Symmetry
}

\author{
Riazuddin \\ National Centre for Physics, \\ Quaid-i-Azam University, \\ Islamabad, Pakistan.
}

December 6, 2018

\begin{abstract}
Phenomenological implications of neutrino oscillations implied by recent experimental data on pattern of neutrino mass matrix are disscussed. It is shown that it is possible to have a neutrino mass matrix which shows approximate flavor symmetry; the neutrino mass differences arise from flavor violation in off-diagonal Yukawa couplings. Two modest extensions of the standard model, which can embed the resulting neutrino mass matix have also been discussed.
\end{abstract}

The data [1, 2, 3, 4, 5] from solar and atmospheric neutrino experiments provide evidence for neutrino mass and mixing. As such lepton flavor is violated providing evidence for physics beyond the standard model (SM). The present experimental data can be explained by neutrino oscillations with mass squared differences and mixing angles having following best fit values [6]

$$
\begin{aligned}
\Delta m_{\text {atm }}^{2} & \equiv \Delta m_{23}^{2}=(2.7 \pm 0.4) \times 10^{-3} \mathrm{eV}^{2} \\
\sin ^{2} \theta_{23} & \equiv \sin ^{2} 2 \theta_{1}=1.00 \pm 0.04 \\
\Delta m_{\text {solar }}^{2} & \equiv \Delta m_{12}^{2}=(7.1 \pm 0.6) \times 10^{-5} \mathrm{eV}^{2} \\
\tan ^{2} \theta_{12} & \equiv \tan ^{2} \theta_{3}=0.45 \pm 0.06
\end{aligned}
$$


Further the CHOOZ [7] experiment gives a bound

$$
\left|U_{e 3}\right|^{2} \equiv \sin ^{2} \theta_{2}<4 \times 10^{-2}
$$

The purpose of this paper is to interpret these results in terms of small offdiagonal perturbations of a degenerate diagonal mass matrix in flavor basis for light Majorana neutrinos. In this approach there is no fundamental distinction between masses of neutrinos of different flavor; the mass differences arise from small flavor violation of off diagonal Yukawa coupling constants. Further it is shown that the neutrino mass differences do not in anyway constraint the absolute value of neutrino mass. The constraint on it will come from neutrinoless double-beta decay experiments, cosmology and direct laboratory experiments, e.g. tritium beta-decay.

Lastly we will try to embed the resulting neutrino mass matrix in two different modest extensions of the standard model. In one version the minimal standard model of particle interactions is extended to include a Higgs triplet to introduce Majorana mass. In the other the standard electroweak gauge group is extended to $S U_{L}(2) \times U_{e}(1) \times U_{\mu}(1) \times U_{\tau}(1)$ leading to off-diagonal mass matrix for light (Majorana) neutrinos which can act as a small perturbation to a degenerate diagonal matrix. The implications of these models on neutrinoless double beta decay are also discussed.

Let us consider a Majorana mass matrix in $(e, \mu, \tau)$ basis

$$
M_{\nu}=m_{0}\left(\begin{array}{ccc}
a_{e e} & a_{e \mu} & a_{e \tau} \\
a_{e \mu} & a_{\mu \mu} & a_{\mu \tau} \\
a_{e \tau} & a_{\mu \tau} & a_{\tau \tau}
\end{array}\right)
$$

It is convenient to define the neutrino mixing angles as follows

$$
\left(\begin{array}{l}
\nu_{e} \\
\nu_{\mu} \\
\nu_{\tau}
\end{array}\right)=U\left(\begin{array}{l}
\nu_{1} \\
\nu_{2} \\
\nu_{3}
\end{array}\right)
$$

where

$$
\left(\begin{array}{ccc}
c_{2} c_{3} & c_{2} s_{3} & s_{2} e^{i \delta} \\
-c_{1} s_{3}-s_{1} s_{2} c_{3} e^{i \delta} & c_{1} c_{3}-s_{1} s_{2} s_{3} e^{i \delta} & s_{1} c_{2} \\
s_{1} s_{3}-c_{1} s_{2} c_{3} e^{i \delta} & -s_{1} c_{3}-c_{1} s_{2} s_{3} e^{i \delta} & c_{1} c_{2}
\end{array}\right)
$$

with $c_{i}=\cos \theta_{i}$ and $s_{i}=\sin \theta_{i}$. We shall put $\delta$ as well as Majorana phases to be zero. In view of Eqs. (11), (2) and (3), we shall take

$$
s_{2}=0, c_{2}=1 \text {. }
$$


The diagonalization of mass matrix (41) give the relations

$$
\begin{aligned}
m_{1} c_{3}^{2}+m_{2} s_{3}^{2} & =a_{e e} \\
m_{1} c_{1}^{2} s_{3}^{2}+m_{2} c_{1}^{2} c_{3}^{2}+m_{3} s_{1}^{2} & =a_{\mu \mu} \\
m_{1} s_{1}^{2} s_{3}^{2}+m_{2} s_{1}^{2} c_{3}^{2}+m_{3} c_{1}^{2} & =a_{\tau \tau} \\
c_{1} s_{3} c_{3}\left(-m_{1}+m_{2}\right) & =a_{e \mu} \\
s_{1} c_{3} s_{3}\left(m_{1}-m_{2}\right) & =a_{e \tau} \\
-c_{1} s_{1}\left(m_{1} s_{3}^{2}+m_{2} c_{3}^{2}-m_{3}\right) & =a_{\mu \tau}
\end{aligned}
$$

Taking now $c_{1}=\frac{1}{\sqrt{2}}, s_{1}=\mp \frac{1}{\sqrt{2}}$, we obtain

$$
\begin{aligned}
& a_{e \mu}= \pm a_{e \tau}=\frac{1}{\sqrt{2}} s_{3} c_{3}\left(-m_{1}+m_{2}\right) \\
& a_{\mu \tau}= \pm \frac{1}{2}\left[\left(m_{1} s_{3}^{2}+m_{2} c_{3}^{2}\right)-m_{3}\right] \\
& a_{\mu \mu}=a_{\tau \tau}=\frac{1}{2}\left(m_{1} s_{3}^{2}+m_{2} c_{3}^{2}+m_{3}\right) \\
& a_{e e}=m_{1} c_{3}^{2}+m_{2} s_{3}^{2}
\end{aligned}
$$

Further defining

$$
\Delta m_{12}^{2}=m_{2}^{2}-m_{1}^{2}, \Delta m_{23}^{2}=m_{3}^{2}-m_{2}^{2}, \Delta m_{13}^{2}=m_{3}^{2}-m_{1}^{2}
$$

so that

$$
\Delta m_{12}^{2}+\Delta m_{23}^{2}=\Delta m_{13}^{2}
$$

and in view of $\Delta m_{12}^{2} \ll \Delta m_{23}^{2}$ as indicated in Eqs. (11), (2), we can take

$$
m_{1} \simeq \pm m_{2}
$$

Thus we have two possibilities for mass matrix $m_{1}=m_{2}=m_{0} ; m_{1}=-m_{2}=$ $m_{0}$ :

$$
\begin{aligned}
& M_{\nu}=m_{0}\left(\begin{array}{ccc}
1 & 0 & 0 \\
0 & \frac{1}{2}(1+a) & \pm \frac{1}{2}(1-a) \\
0 & \pm \frac{1}{2}(1-a) & \frac{1}{2}(1+a)
\end{array}\right) \\
& M_{\nu}=m_{0}\left(\begin{array}{ccc}
\cos 2 \theta_{3} & -\frac{1}{\sqrt{2}} \sin 2 \theta_{3} & \mp \frac{1}{\sqrt{2}} \sin 2 \theta_{3} \\
-\frac{1}{\sqrt{2}} \sin 2 \theta_{3} & \frac{1}{2}\left(\cos 2 \theta_{3}+a\right) & \pm \frac{1}{2}\left(\cos 2 \theta_{3}-a\right) \\
\mp \frac{1}{\sqrt{2}} \sin 2 \theta_{3} & \pm \frac{1}{2}\left(\cos 2 \theta_{3}-a\right) & \frac{1}{2}\left(\cos 2 \theta_{3}+a\right)
\end{array}\right)
\end{aligned}
$$


where $a=m_{3} / m_{0}$. If we do not want to commit to any particular value of $\theta_{3}$, then we have the first case with the following subcases corresponding to $m_{0}=0, a=0, a=-1,1,-2,2$ respectively

$$
\begin{array}{cl}
\text { i) }: & \frac{m_{3}}{2}\left(\begin{array}{lll}
0 & 0 & 0 \\
0 & 1 & 1 \\
0 & 1 & 1
\end{array}\right) \\
\text { ii) }: & m_{0}\left(\begin{array}{lll}
1 & 0 & 0 \\
0 & 0 & 1 \\
0 & 1 & 0
\end{array}\right) \\
\text { iii) }: & m_{0}\left(\begin{array}{lll}
1 & 0 & 0 \\
0 & 1 & 0 \\
0 & 0 & 1
\end{array}\right) \\
\text { iv) }: & \frac{m_{0}}{2}\left(\begin{array}{ccc}
2 & 0 & 0 \\
0 & -1 & 3 \\
0 & 3 & -1
\end{array}\right) \\
\text { v) }: & \frac{m_{0}}{2}\left(\begin{array}{ccc}
2 & 0 & 0 \\
0 & 3 & -1 \\
0 & -1 & 3
\end{array}\right)
\end{array}
$$

In order to generate $\Delta m_{12}^{2}$ and $\Delta m_{23}^{2}$, we will now concentrate on choice (iii) which preserves flavor and add to it a small perturbation which violates flavor in off-diagonal matrix elements:

$$
M_{\nu}=m_{0}\left(\begin{array}{ccc}
1 & \varepsilon_{12} & \varepsilon_{13} \\
\varepsilon_{12} & 1 & \varepsilon_{23} \\
\varepsilon_{13} & \varepsilon_{23} & 1
\end{array}\right)
$$

where $\varepsilon_{i j} \ll 1$. The diagonalization gives

$$
m_{i}=m_{0}\left(1-x_{i}\right)
$$

where $x_{i}(i=1,2,3)$ are roots of cubic equation

$$
x^{3}-\left(\varepsilon_{12}^{2}+\varepsilon_{13}^{2}+\varepsilon_{33}^{2}\right) x+2 \varepsilon_{12} \varepsilon_{13} \varepsilon_{23}=0
$$

The choice $\varepsilon_{12}=\varepsilon_{13}=\varepsilon_{23}=\varepsilon$ will give the roots $(\varepsilon, \varepsilon,-2 \varepsilon)$ and thus will not lift the degeneracy between $m_{1}$ and $m_{2}$. To lift this degeneracy we take 
$\varepsilon_{12}=\varepsilon_{13}=\varepsilon+\delta, \varepsilon_{23}=\varepsilon$ with $\delta / \varepsilon \ll 1$. Then the roots to the first order in $\delta / \varepsilon$ are $\varepsilon, \varepsilon\left(1+\frac{4}{3} \delta / \varepsilon\right),-2 \varepsilon\left(1+\frac{2}{3} \delta / \varepsilon\right)$ so that

$$
\begin{aligned}
m_{1} & =m_{0}\left[1-\varepsilon-\frac{4}{3} \delta\right] \\
m_{2} & =m_{0}[1-\varepsilon] \\
m_{3} & =m_{0}\left[1+2 \varepsilon+\frac{4}{3} \delta\right] \\
\Delta m_{12}^{2} & \approx \frac{8}{3} m_{0}^{2} \delta(1-\varepsilon) \simeq \frac{8}{3} m_{0}^{2} \delta \\
\Delta m_{32}^{2} & \approx 6 \varepsilon m_{0}^{2}
\end{aligned}
$$

This gives

$$
\begin{gathered}
\frac{\delta}{\varepsilon}=\frac{9}{4} \frac{\Delta m_{12}^{2}}{\Delta m_{32}^{2}} \simeq 5.9 \times 10^{-2} \\
\sqrt{\varepsilon} m_{0} \simeq 2.1 \times 10^{-2} \mathrm{eV}
\end{gathered}
$$

so that $m_{0}$ is not constrained. However, $m_{0}$ is constrained by WMAP data [10, $3 m_{0}<0.71 \mathrm{eV}$. When analyzed in conjunction with neutrino oscillation, it is found that mass eigenvalues are essentially degenerate with $3 m_{0}>0.4$ $\mathrm{eV}$ [1] The above limits put limits on $\varepsilon: 7.9 \times 10^{-3}<\varepsilon<2.5 \times 10^{-2}$.

It is pertinent to remark that in view of small mass differences involved [cf. Eqs. (1) and (2)], and that analysis of WMAP data in conjunction with neutrino oscillation imply essentially degenrate mass eigenvalues, it is quite natural to remove the degenracy by small perturbations. Such a procedure should be stable as the parameter $\varepsilon$ turns out to be small in view of limit on it given above. The $\delta / \varepsilon \simeq 6 \times 10^{-2}$ may indicate fine tuning but this a problem common to Yukawa couplings even in charged sector, the solution of which has so far evaded us.

Next we consider an extension of the standard electroweak model to

$$
G \equiv S U_{L}(2) \times U_{e}(1) \times U_{\mu}(1) \times U_{\tau}(1)
$$

which naturally gives an off diagonal mass matrix for light neutrinos [12]. In addition to usual fermions $L_{i}=\left(\begin{array}{c}\nu^{i} \\ e^{i}\end{array}\right)_{L}, e_{R}^{i}$, there are three right handed $S U_{L}(2)$-singlet neutrinos $N_{R}^{i}$ which carry $U_{i}(1)$ quantum numbers $(-1,1,0)$, 
$(1,0,-1)$ and $(0,-1,1)$. Further in addition to $S U_{L}(2)$ Higgs doublets, there are three Higgs $S U_{L}(2)$ singlets $\Sigma^{i}$ with $U_{i}(1)$ quantum numbers $(1,-1,0)$, $(1,0,-1)$ and $(0,1,-1)$. In order to introduce diagonal terms we also introduce a right handed neutrino $N$ and a corresponding Higgs boson $\Sigma$ which are $S U_{L}(2)$ and $U_{i}(1)$ singlets. The symmetry is spontaneously broken by giving vacuum expectation values to Higgs boson $\phi^{(i)}, \Sigma^{(i)}$ and $\Sigma$ :

$$
\left\langle\phi^{(i)}\right\rangle=\frac{v_{i}}{\sqrt{2}},\left\langle\Sigma^{(i)}\right\rangle=\frac{\Lambda_{i}}{\sqrt{2}},\langle\Sigma\rangle=\frac{\Lambda}{\sqrt{2}}
$$

where for simplicity we shall take $v_{1}=v_{2}=v_{3}=v$ and $\Lambda_{1}=\Lambda_{2}=\Lambda_{3}=\Lambda$ (any difference can be absorbed in the corresponding Yukawa couplings of fermions with Higgs bosons). The resulting mass matrix through seesaw mechanism for light neutrinos is

$$
M_{\nu}=\frac{v^{2}}{2 M_{R}}\left(\begin{array}{ccc}
h_{1}^{(1)} h_{1}^{(1)} & h_{1}^{(2)} h_{2}^{(3)} & h_{1}^{(2)} h_{3}^{(1)} \\
h_{1}^{(2)} h_{2}^{(3)} & h_{2}^{(2)} h_{2}^{(2)} & h_{2}^{(3)} h_{3}^{(1)} \\
h_{1}^{(2)} h_{3}^{(1)} & h_{2}^{(3)} h_{3}^{(1)} & h_{3}^{(3)} h_{3}^{(3)}
\end{array}\right)
$$

where $M_{R}=\frac{f \Lambda}{\sqrt{2}}$. We now put $h_{i}^{(i)}=h, \frac{h_{1}^{(2)} h_{2}^{(3)}}{h^{2}}=\varepsilon_{12}, \frac{h_{1}^{(2)} h_{3}^{(1)}}{h^{2}}=\varepsilon_{13}, \frac{h_{2}^{(3)} h_{3}^{(1)}}{h^{2}}=$ $\varepsilon_{23}$. This gives the desired matrix (9) with $m_{0}=\frac{h^{2} v^{2}}{2 M_{R}}$. If $h$ is of the order of 1 and $m_{0}$ is few electron volts, then one requires $M_{R} \simeq 10^{13} \mathrm{GeV}$.

Another simple way to obtain the neutrino mass matrix (9) is to include a Higgs triplet $\Delta(3,2):\left(\Delta^{++}, \Delta^{+}, \Delta^{0}\right)$ with the interaction [13, 14]

$$
\mathcal{L}=h_{i j} L_{i}^{T} i \tau_{2}(\vec{\tau} \cdot \vec{\Delta}) L_{j}
$$

generating neutrino mass matrix

$$
\left(M_{\nu}\right)_{i j}=2 h_{i j}\left\langle\Delta^{0}\right\rangle
$$

Taking $h_{11}=h_{22}=h_{33}=h$ and $\frac{h_{12}}{h}=\varepsilon_{12}, \frac{h_{13}}{h}=\varepsilon_{13}$, and $\frac{h_{23}}{h}=\varepsilon_{23}$; $m_{0}=2 h\left\langle\Delta^{0}\right\rangle$ we get the desired mass matrix. If $h$ is of order unity, and $m_{0}$ is a few electron volts, then $\left\langle\Delta^{0}\right\rangle$ is of order of eV, which appears to be unnatural. It is well known that the coupling of $\Delta$ to gauge field will alter the $\rho$ parameter $\left(\rho \equiv \frac{m_{w}^{2}}{m_{z}^{2} \cos ^{2} \theta_{w}}\right)$ by

$$
\Delta \rho \equiv(1-\rho)=4\left\langle\Delta^{0}\right\rangle^{2} / v^{2}
$$


The present limit on $\Delta \rho(\leq 0.02)$ only requires $\left\langle\Delta^{0}\right\rangle<25 \mathrm{GeV}$. To give a more natural look to small value of $\Delta^{0}$, lepton flavor violating term of the form $\mu\left(\phi^{\dagger} \Delta \tilde{\phi}\right)+H . C$ is added to $L$ conserving Higgs potential. Thus [14]

$$
\left\langle\Delta^{0}\right\rangle=\frac{\mu v^{2}}{2 M_{\Delta}^{2}} \simeq 3 \times 10^{4} \mu\left(\frac{G e V^{2}}{M_{\Delta}^{2}}\right)
$$

For $\left\langle\Delta^{0}\right\rangle \sim \mathrm{eV} \mu$ has to be quite small $(\simeq 3 \mathrm{eV})$ if $M_{\Delta}$ is $\sim 1 \mathrm{TeV}$. If $\mu \sim$ $1 \mathrm{GeV}, M_{\Delta} \simeq 3 \times 10^{6} \mathrm{GeV}$.

Finally we discuss the implications of above models in neutrinoless double beta decay. In the first model at tree level there is only the standard contribution through light Majorana $\nu_{e}$. In the second model, there could be additional contribution at tree level through diagram in figure 1.

This give rise to an effect $\Delta L=2$ interaction which can be written as 15

$$
\begin{array}{r}
H_{\Delta L=2}=-G_{F}^{2} \epsilon_{1}^{e e} \bar{u}\left(1+\gamma_{5}\right) d \bar{u} \gamma_{\mu}\left(1-\gamma_{5}\right) d \\
\bar{e} \gamma^{\mu}\left(1-\gamma_{5}\right) \frac{1}{\gamma-q} C \bar{e}^{T}
\end{array}
$$

where $\left[h_{11}=h\right]$

$$
\epsilon_{1}^{e e}=\frac{h_{u}\left(h \mu \frac{v}{\sqrt{2}}\right)}{u \sqrt{2} G_{F} m_{\Delta}^{2} m_{H}^{2}}
$$

On using Eq. (20) and that $m_{0}=2 h\left(\Delta^{0}\right)$, we obtain

$$
\epsilon_{1}^{e e}=\frac{\left(h_{u}\right) m_{0}}{8 \sqrt{2} G_{F} m_{H}^{2} \frac{v}{\sqrt{2}}}
$$

With $\frac{v}{\sqrt{2}} \simeq 175 \mathrm{GeV}$, we obtain $\epsilon_{1}^{e e}=h_{u}\left(\frac{m_{0}}{e V}\right)\left(5 \times 10^{-10}\right)$. One may expect [15] $h_{u}=m_{u} / m_{W} \simeq 5 \times 10^{-5}$ so that $\epsilon_{1}^{e e}$ is much too small compared to the upper limit on $\epsilon_{1}^{e e}\left(\leq 10^{-8}\right)$ found in [15] from the neutrino mass contribution to $\beta \beta_{0} \nu\left(m_{\nu}<1 \mathrm{eV}\right)$.

To sum up we have shown that it is possible to interpret the neutrino mass differences and mixings with approximate flavor symmetry. The small flavor violation, manifested in neutrino mass differences, is in the Yukawa coupling constants $h_{i j}$ which determine the off-diagonal matrix elements with $\varepsilon_{i j}(i \neq j) \equiv \frac{h_{i j}^{2}(i \neq j)}{h^{2}} \sim 10^{-3}$. This may have some phenomenological implications. We have also discussed two modest extensions of the standard model which can embed the resulting neutrino mass matrix. 
Note added: It has been called to my attention by A.Zee that the matrix(9) in this paper is similar to $\widetilde{M}_{0}$ with $y=-1$, given by X.G.He and A.Zee [16]. Our motivation is rather different.

\section{Acknowledgement}

This work was supported by the grant from Pakistan Council of Science and Technology.

\section{References}

[1] K. Hagiwara et al., (Particle Data Group) Phys. Rev. D 66, 010001 (2002)

[2] Q. R. Ahmad et al., (SNO collaboration), Phys. Rev. Lett. 89, 011301 (2002); Phys. Rev. Lett. 89011302 (2002); S. Fukada et. al., (SuperKamiokande Collaboration), Phys. Lett. B 539 , 179(2002); B. T. Cleveland et al., Astrophys. J. 496, 505 (1998); R. Davis, Prog. Part. Nucl Phys. 32, 13(1994); D. N. Abdurashitov et al., (SAGE Collaboration), Phys. Rev. D60, 055801(1999); arXiv: astro-ph/0204205. W. Hampel at al., (GALLEX Collaboration), Phys. Lett. B447, 127(1999); C. Cattadori, (GNO Collaboration), Nucl. Phys. B111 (Proc. Suppl.), $311(2002)$.

[3] Y. Fukuda et al., (Kamiokande Collaboration), Phys. Lett. B335, 237(1994); R. Becker-Szendy et al., (IBM Collaboration), Nucl. Phys. B38 (Proc. Suppl.), 331(1995); W. W. M. Allison et al., (Soudan Collaboration), Phys. Lett. B449, 137(1999); M. Ambrosio et al., (MACRO Collaboration) Phys. Lett. B434, 451(1998); M. Soili, arXiv: hep-ex/0211030.

[4] K. Eguchi et al., (KamLAND Collaboration), arXiv: hep-ph/0212021.

[5] M. H. Ahn et al., (K2K Collaboration), arXiv: hep-ph/0212007.

[6] See e.g. in the proceedings of the $\mathrm{X}$ international worshop on neutrino telescopes, Venezia, march 11-14, 2003. Transparencies are available at the internet address axpd24.pd.infn.it/conference2003/venice03. html.

[7] M. Apollonio et. al., (CHOOZ Collaboration), Phys. Lett. 466, 415(1999) [arXiv: hep-ph/9907037. 
[8] S. F. King, arXiv: hep-ph/0210081.

[9] Xiao-Gang He and A. Zee, arXiv:hep-ph/0302201.

[10] D.N.Spergel et al, First year Wilkinson Microwave Anisotropy Probe (WMAP) Observations: Determination of Cosmological Parameters, arXiv: astro-ph/0302209.

[11] O.Elgasey and O.Lahav, Upper Limits on Neutrino Masses from the 2dFGRS and WMAP: the Role of Priors, arXiv: astro-ph/0303089V2.

[12] Fayyazuddin and Riazuddin, Phys. Rev. D 35 2201(1987); Riazuddin, Phys. Rev. D63, 033003(2001).

[13] J. Schechter and J. W. F. Valle, Phys. Rev. D22, 2227(1980); Riazuddin, R. E. Marshak and R. N. Mohapatra, Phys. Rev. D24, 1310(1981); Ernest Ma., Martti Raidal, and Utpal Sarkar, Phys. Rev. Lett. 85, 3769 (2000).

[14] E. Ma, arXiv: hep-ph/0204013.

[15] K.S. Babu and R. N. Mohapatra, New vector-scalar contribution to neutrinoless double beta decay and constraints on R-parity violation, Phys. Rev. Lett. 75, 2276 (1995)

[16] Xiao-Gang He and A.Zee, Some Simple Mixing and Mass Matrices for Neutrinos hep-ph/0301092;A.Zee,Some Remarks on Neutrino Oscillation and Masses, arxiv:hep-ph/0307155

\section{Figure Caption:}

Figure 1: The vector-scalar exchange diagram for $\beta \beta_{0 \nu}$ 
This figure "figr.jpg" is available in "jpg" format from: http://arxiv.org/ps/hep-ph/0307347v2 